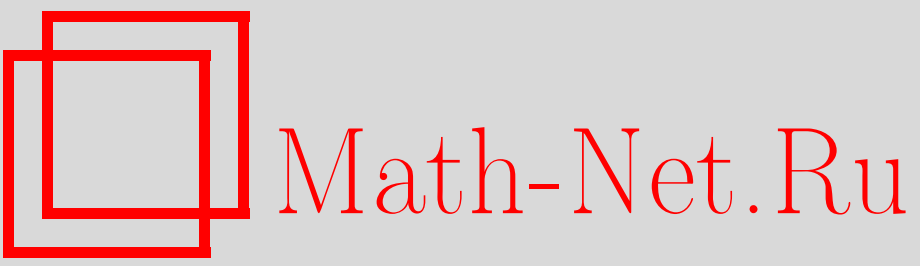

О. И. Мохов, Интегрируемые бигамильтоновы иерархии, порождаемые согласованными метриками постоянной римановой кривизны, УМH, 2002, том 57, выпуск 5, 157-158

DOI: https://doi.org/10.4213/rm563

Использование Общероссийского математического портала Math-Net.Ru подразумевает, что вы прочитали и согласны с пользовательским соглашением

http: //www . mathnet.ru/rus/agreement

Параметры загрузки:

IP : 54.164 .48 .24

26 апреля 2023 г., 18:21:25

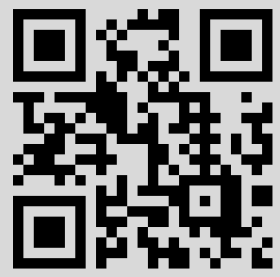




\title{
ИНТЕГРИРУЕМЫЕ БИГАМИЛЬТОНОВЫ ИЕРАРХИИ, ПОРОЖДАЕМЫЕ СОГЛАСОВАННЫМИ МЕТРИКАМИ ПОСТОЯННОЙ РИМАНОВОЙ КРИВИЗНЫ
}

\author{
О.И. Мохов
}

Построены интегрируемые бигамильтоновы иерархии, порождаемые согласованными метриками постоянной кривизны. Две контравариантные метрики $g_{1}^{i j}(u)$ и $g_{2}^{i j}(u)$ постоянной римановой кривизны $K_{1}$ и $K_{2}$ соответственно называются согласов анными, если любая линейная комбинация этих метрик $g^{i j}(u)=\lambda_{1} g_{1}^{i j}(u)+\lambda_{2} g_{2}^{i j}(u)$ является метрикой постоянной римановой кривизны $\lambda_{1} K_{1}+\lambda_{2} K_{2}$, а коэффициенты соответствующих связностей Леви-Чивиты связаны соотношением $\Gamma_{k}^{i j}(u)=\lambda_{1} \Gamma_{1, k}^{i j}(u)+\lambda_{2} \Gamma_{2, k}^{i j}(u)$ [1]. Задача описания согласованных метрик постоянной римановой кривизны эквивалентна задаче описания согласованных нелокальных гамильтоновых операторов гидродинамического типа, порождаемых метриками постоянной римановой кривизны (согласованных гамильтоновых операторов Мохова-Ферапонтова [2]). Как показано в [3], [4] (см. также [1]), согласованные метрики постоянной римановой кривизны описьваются совместной нелинейной системой, интегрируемой методом обратной задачи. В данной статье по любому решению этой интегрируемой системы, т.е. для любой пары согласованных метрик постоянной римановой кривизны, построены в явном виде интегрируемые бигамильтоновы системы, обладающие соответствующей парой согласованных гамильтоновых операторов. В плоском случае $\left(K_{1}=K_{2}=0\right)$, для согласованных гамильтоновых операторов Дубровина-Новикова [5], эта задача была рассмотрена и полностью решена в работах автора [6], [7]. В [2] были введены и изучены нелокальные гамильтоновы операторы, имеющие следующий вид (гамильтоновы операторы Мохова-Ферапонтова):

$$
P^{i j}=g^{i j}(u(x)) \frac{d}{d x}+b_{k}^{i j}(u(x)) u_{x}^{k}+K u_{x}^{i}\left(\frac{d}{d x}\right)^{-1} u_{x}^{j} .
$$

Если $\operatorname{det}\left(g^{i j}(u)\right) \not \equiv 0$ (такие операторы вида (1) назьваются невырожсденными), то оператор (1) является гамильтоновьг тогда и только тогда, когда $g^{i j}(u)$ - произвольная контравариантная метрика постоянной римановой кривизны $K, b_{k}^{i j}(u)=-g^{i s}(u) \Gamma_{s k}^{j}(u)$, где $\Gamma_{s k}^{j}(u)$ - связность, порождаемая метрикой $g^{i j}(u)$ (связность Леви-Чивиты) [2]. В задаче об описании произвольной пары согласованных нелокальных гамильтоновых операторов вида (1) один из этих гамильтоновых операторов всегда можно считать локальным (т.е. для него $K=0$ ) без ограничения общности. Рассмотрим задачу о согласованности нелокального и локального гамильтоновых операторов вида (1). По теореме Дубровина-Новикова [5] для любого невырожденного локального гамильтонова оператора вида (1) с $K=0$ всегда существуют локальные координаты $u^{1}, \ldots, u^{N}$ (плоские координаты соответствующей метрики), в которых этот оператор имеет вид $P_{2}^{i j}=\eta^{i j}(d / d x)$, где $\eta^{i j}=\eta^{j i}, \eta^{i j}=$ const, $\operatorname{det} \eta^{i j} \neq 0$.

ТеОРема 1. Произвольныци нелокальньй гамильтонов оператор $P_{1}^{i j}$ вида (1) (возможно, вырожденный) согласован с гамильтоновым оператором $P_{2}^{i j}=\eta^{i j}(d / d x)$ тогда и только тогда, когда он имеет вид

$$
P_{1}^{i j}=\left[\eta^{i s} \frac{\partial H^{j}}{\partial u^{s}}+\eta^{j s} \frac{\partial H^{i}}{\partial u^{s}}-K u^{i} u^{j}\right] \frac{d}{d x}+\left[\eta^{i s} \frac{\partial^{2} H^{j}}{\partial u^{s} \partial u^{k}}-K \delta_{k}^{i} u^{j}\right] u_{x}^{k}+K u_{x}^{i}\left(\frac{d}{d x}\right)^{-1} u_{x}^{j},
$$

где $H^{i}(u), 1 \leqslant i \leqslant N,-$ гладжие функции, определенные в некоторой области локальных координат.

В плоском случае $(K=0)$ соответствуюшее утверждение было сформулировано автором в [8], [9] (см. также условия на плоские пучки метрик в [10]).

ТЕОРема 2. Нелокальный оператор (2) (возможно, вырожденный) является гамильтоновым тогда и только тогда, когда выполнены следующие уравнения:

Работа вьполнена при финансовой поддержке Фонда Александра фон Гумбольта (Германия), а также РФФИ (грант № 02-01-00803) и фонда INTAS (грант № 99-1782). 


$$
\begin{gathered}
\frac{\partial^{2} H^{i}}{\partial u^{k} \partial u^{s}} \eta^{s p} \frac{\partial^{2} H^{j}}{\partial u^{p} \partial u^{l}}=\frac{\partial^{2} H^{j}}{\partial u^{k} \partial u^{s}} \eta^{s p} \frac{\partial^{2} H^{i}}{\partial u^{p} \partial u^{l}} \\
\left(\eta^{i r} \frac{\partial H^{s}}{\partial u^{r}}+\eta^{s r} \frac{\partial H^{i}}{\partial u^{r}}-K u^{i} u^{s}\right) \eta^{j p} \frac{\partial^{2} H^{k}}{\partial u^{p} \partial u^{s}}=\left(\eta^{j r} \frac{\partial H^{s}}{\partial u^{r}}+\eta^{s r} \frac{\partial H^{j}}{\partial u^{r}}-K u^{j} u^{s}\right) \eta^{i p} \frac{\partial^{2} H^{k}}{\partial u^{p} \partial u^{s}} .
\end{gathered}
$$

В плоском случае $(K=0)$ соответствующая теорема получена автором в [9], где была сформулирована также и гипотеза об интегрируемости системы $(3),(4)$ методом обратной задачи рассеяния, доказанная впоследствии в работах автора [11], [12], [1] (см. также [13], где найдена пара Лакса). Соответствующие общие условия на плоские пучки метрик были указаны в [10].

Tеорема 3. Система (3), (4) интегрируется методом обратной задачи.

Рассмотрим оператор рекурсии $R=P_{1}\left(P_{2}\right)^{-1}$, задаваемый каноническими согласованными гамильтоновыми операторами $P_{2}^{i j}=\eta^{i j}(d / d x)$ и $P_{1}^{i j}(2)$. Любая система из иерархии $u_{t_{n}}^{i}=$ $\left(R^{n}\right)_{j}^{i} u_{x}^{j}, n \in \mathbb{Z}$, является мултигамильтоновой интегрируемой системой. В частности, интегрируемой является любая система вида $u_{t_{1}}^{i}=R_{j}^{i} u_{x}^{j}$, т.е. система гидродинамического типа

$$
u_{t_{1}}^{i}=\left(h^{i}(u)+\eta^{i s} \frac{\partial h^{j}}{\partial u^{s}} \eta_{j l} u^{l}-\frac{K}{2} \eta_{s k} u^{i} u^{s} u^{k}\right)_{x},
$$

где $h^{i}(u), 1 \leqslant i \leqslant N,-$ произвольное решение интегрируемой системы (3), (4); $\eta^{i s} \eta_{s j}=\delta_{j}^{i}$. Эта система гидродинамического типа бигамилтонова с парой канонических согласованных гамильтоновых операторов $P_{2}^{i j}=\eta^{i j}(d / d x)$ и $P_{1}^{i j}(2)$ :

$$
\begin{aligned}
& u_{t_{1}}^{i}=P_{1}^{i j} \frac{\delta H_{1}}{\delta u^{j}(x)}, H_{1}=\frac{1}{2} \int \eta_{j l} u^{j}(x) u^{l}(x) d x, \\
& u_{t_{1}}^{i}=P_{2}^{i j} \frac{\delta H_{2}}{\delta u^{j}(x)}, H_{2}=\int\left(\eta_{j k} h^{k}(u(x)) u^{j}(x)-\frac{K}{8} \eta_{j k} \eta_{s l} u^{j}(x) u^{k}(x) u^{s}(x) u^{l}(x)\right) d x .
\end{aligned}
$$

Следующей системой в иерархии (при $n=2$ ) является интегрируемая система

$$
\begin{aligned}
u_{t_{2}}^{i}= & \left(( \eta ^ { i s } \frac { \partial h ^ { j } } { \partial u ^ { s } } + \eta ^ { j s } \frac { \partial h ^ { i } } { \partial u ^ { s } } - K u ^ { i } u ^ { j } ) \left(\eta_{j l} \frac{\partial h^{l}}{\partial u^{k}}+\eta_{r k} \frac{\partial h^{r}}{\partial u^{j}}+\eta_{r q} u^{q} \frac{\partial^{2} h^{r}}{\partial u^{j} \partial u^{k}}-K \eta_{j l} \eta_{p k} u^{l} u^{p}\right.\right. \\
& \left.-\frac{K}{2} \eta_{j k} \eta_{p l} u^{l} u^{p}\right)+\left(\eta^{i s} \frac{\partial^{2} h^{j}}{\partial u^{s} \partial u^{k}}-K \delta_{k}^{i} u^{j}\right)\left(\eta_{j l} h^{l}(u)+\eta_{r q} u^{q} \frac{\partial h^{r}}{\partial u^{j}}\right. \\
& \left.\left.-\frac{K}{2} \eta_{j l} \eta_{p r} u^{l} u^{p} u^{r}\right)+K \delta_{k}^{i}\left(\eta_{j l} h^{l}(u) u^{j}-\frac{K}{8} \eta_{j l} \eta_{p r} u^{j} u^{l} u^{p} u^{r}\right)\right) u_{x}^{k}
\end{aligned}
$$

\section{СПИСОК ЛИТЕРАТУРЫ}

[1] О. И. Мохов // Функц. анализ и его прил. 2001. Т. 35. № 2. C. 24-36; math.DG/0005051. [2] О. И. Мохов, Е. В. Ферапонтов // УМН. 1990. Т. 45. № 3. С. 191-192. [3] О. И. Мохов // math.DG/0201280. [4] О.И. Мохов // УМН. 2002. Т. 57. №3. С. 155-156. [5] Б. А. Дубровин, С. П. Новиков // Докл. АН СССР. 1983. Т. 270. № 4. С. 781-785. [6] О. І. Mokhov // Proceedings of the International Conference "Nonlinear Evolution Equations and Dynamical Systems", Cambridge (England), July 24-30, 2001; math.DG/0201281. [7] О.И. Мохов // УМН. 2002. Т. 57. № 1. С. 157-158. [8] О. И. Мохов // УМН. 1997. Т. 52. №6. С. 171-172. [9] О. И. Мохов // Труды МИРАН. 1999. Т. 225. С. 284-300. [10] B. Dubrovin // Lecture Notes in Math. 1996. V. 1620. Р. 120-348; hep-th/9407018 (1994). [11] О. И. Мохов // ТМФ. 2002. Т. 130. № 2. С. 233-250; math.DG/0005081. [12] О. И. Мохов // УМН. 2001. Т. 56. № 2. C. 221-222. [13] E. V. Ferapontov // math.DG/0005221.

Центр нелинейньх исследований при Институте теоретической физики им. Л. Д. Ландау РАН 